\title{
Growth-related profiles of remanent flux in bulk melt-textured YBaCuO crystal magnetized by pulsed fields
}

\author{
A B Surzhenko††, S Schauroth $\dagger$, D Litzkendorf $\dagger$, \\ M Zeisberger $\dagger$, T Habisreuther $\dagger$ and $\mathrm{W}$ Gawalek $\dagger$ \\ † Institut für Physikalische Hochtechnologie, Winzerlaer Str. 10, Jena D-07745, \\ Germany \\ $\ddagger$ Institute for Magnetism, Vernadsky Str. 36b, Kyiv 03142, Ukraine
}

\begin{abstract}
We have studied the remanent magnetic flux distribution in bulk melttextured $\mathrm{YBa}_{2} \mathrm{Cu}_{3} \mathrm{O}_{7-\delta}(\mathrm{YBCO})$ crystals after their magnetization in quasi-static and pulsed magnetic fields up to $6 \mathrm{~T}$. It has been shown that, provided that the magnetic pulse is sharp enough and its amplitude much exceeds the twice penetration magnetic field, the pulse magnetization technique becomes extremely sensitive to the sample inhomogeneities. Using this method with appropriate parameters of the magnetic pulse, we have particularly demonstrated that the growth of YBCO crystals in the growth sectors responds for a macroscopic arrangement of weaks links.

PACS numbers: $74.72 \mathrm{Bk}, 74.80 \mathrm{Bj}$
\end{abstract}

\section{Introduction}

The recent progress in melt processing enables to fabricate large $\mathrm{RE}-\mathrm{Ba}-\mathrm{Cu}-\mathrm{O}$ ( $\mathrm{RE}$ is the rare-earth element) high-temperature superconductors (HTS) that look very promising for manifold applications, viz., for HTS permanent magnets, magnetic bearings and flywheels, electromotors and generators, current limiters, etc. [1] Thus, it is vitally important to find fast, sensitive and non-destructive method(s) for the quality tests of the HTS material.

Now the remanent magnetic flux profiles [2, 3] of the field-cooled (FC) or zero-fieldcooled (ZFC) samples are prevalent for these tasks. Compared to permanent magnets [3], strong magnetic fields of superconducting coils result in an increased sensitivity to the sample inhomogeneities. However, this seems to be essentially improved with the pulsed field magnetization (PFM). Till nowadays this technique was mainly considered as a convenient method to magnetize the HTS permanent magnets if these are preinstalled in various equipment such as, for example, electromotors [4]. The main problem, which was encountered by various authors [5 9], consisted in the trapped flux losses related to the sample heating by viscous forces that exert on moving flux lines [10. Thus, the main pains were taken to reduce this destructive effect. In particular, various 
parameters of magnetic pulses (i.e. the shapes, durations and amplitudes) [5 77 and/or their sequences [8, 9] were tested for these purposes. However, being harmful for practical magnetization of HTS bulks, this phenomenon promises a certain profit for their characterization. In fact, the local areas containing weak links have been shown to be more heated than those where weak links are absent [5]. If the magnetic pulse is short enough, the heat transfer during the pulse is nearly negligible. In this case, the temperature distribution just after the pulse has to correlate with the material quality and yet more enlarge the contrast between the magnetic flux trapped by areas with and without weak links. Thus, the recipe of how to increase this contrast seems simple: one has to accelerate the magnetic flux motion and to shorten the pulse duration. But despite of evident benefits for the HTS sample characterization, the PFM method did not gain a respective reputation so far. Moreover, no detailed studies: Which parameters of the magnetic pulse are enough to provide the required sensibility? were performed.

Meantime, a macroscopic arrangement of structural imperfections (e.g., subgrains and 211-inclusions) which is related to the growth of the HTS crystals was recently inquired by polarized light methods [11]. Due to extremely short coherence length $\xi$ which is typical for HTSs, this arrangement may certainly be expected to result in the growth-related magnetic properties. Except for an apparent importance of this feature itself, its detection by the PFM technique could serve as the best criterion whether a necessary sensitivity is already attained. To our knowledge, no growth-related profiles of the remanent magnetic flux were reported hitherto. That is what we are going to demonstrate in the current paper.

\section{Experimental details}

To avoid unwanted consequences that could be induced by cracks and other macroscopic imperfections, we have knowingly selected rather homogeneous $\mathrm{YBa}_{2} \mathrm{Cu}_{3} \mathrm{O}_{7-\delta}$ (YBCO) sample (Figure 1). This was sinthesized from the mixture of commercially purchased powders (Solvay GmbH, Germany) with 1 wt. $\% \mathrm{CeO}_{2}$ by the melt-processing technique 12] and post-annealed in oxygen atmosphere. The bulk thus prepared was a cylinder with a diameter of $30 \mathrm{~mm}$ and a thickness of $18 \mathrm{~mm}$. To reduce a plausible scatter of flux distribution caused by a roughness of the top surface, the sample was polished.

The optical image of the polished surface (see Figure —(a)) clearly exhibited a trace in its geometric centre, i.e. under the point where the $\mathrm{SmBa}_{2} \mathrm{Cu}_{3} \mathrm{O}_{X}$ seed was placed, and the X-like cross separating the crystal onto four 90-degree segments. These features correspond to five growth sectors (GSs), i.e. areas grown on different habit planes: the c-GS with the habit (001) perpendicular to the c-axis, [001], and four aGSs with the (100), (010), (100) and (010) habit planes (see the schematic illustration in Figure 1(c)) [1]. In other words, both the central trace and the cross display the GS boundaries (GSBs), viz., a-c-GSBs and a-a-GSBs, respectively, which are pathways of crystal edges between two neighboring habits during the growth stage. Although a regular shape of the remanent flux map presented in Figure 1 (b) well confirmed the 

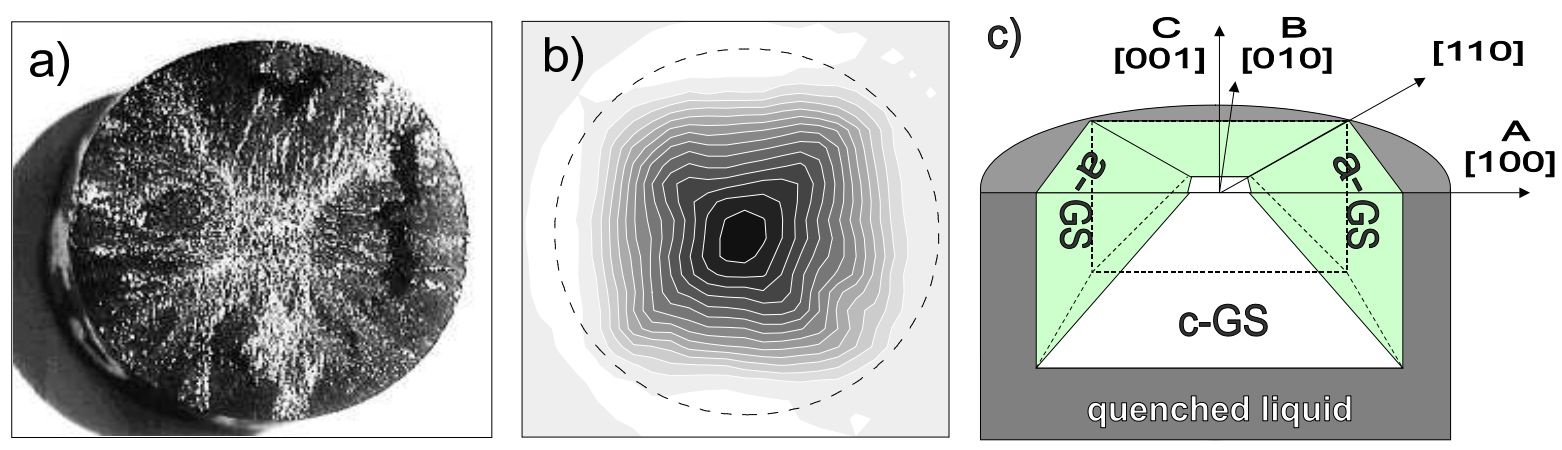

Figure 1. The bulk YBCO sample that was studied in the current work. The $90^{\circ}$ cross on (a) the optical image and nearly regular tetragonal shape of isolines on (b) the remanent magnetic flux map ( $\mathrm{FC}$ magnetization at $\mathrm{T}=77 \mathrm{~K}$ and $\mu_{0} H=1 \mathrm{~T}$, peak value $\left.B_{\max }=0.63 \mathrm{~T}\right)$ testify to the homogeneity of the crystal structure which sketch is illustrated in figure (c).

sample homogeneity, we shall hereafter report in what devastating contrast may minor inhomogeneities result under the PFM.

In order to magnetize the sample we used either quasi-static fields with a sweep rate $V=\mu_{0} d H / d t \simeq 0.01 T / s$ or the sin-wave-shaped magnetic pulses $(V \leq 6000 \mathrm{~T} / \mathrm{s})$. In all cases, the sample was magnetized in the vertical direction, i.e. along the c-axis, [001]. The static magnetization (both in the FC and ZFC modes) was provided by the iron-yoke magnet $\left(\mu_{0} H \leq 1 T\right)$ and the superconducting solenoid $\left(\mu_{0} H \leq 8 T\right)$. Magnetic pulses were supplied by discharging of capacitors (with a total capacity of $5 \mathrm{mF}$ ) charged up to $2 \mathrm{kV}$ through a circuit consisting of a copper coil and a thyristor which flashed only the first half $(0 \leq \omega t \leq \pi)$ of a sin-wave $H=H^{*} \sin (\omega t)$. This form, amplitude $\left(\mu_{0} H^{*} \leq 6 T\right)$ and duration $\left(t_{\max }=\pi / \omega=3 \mathrm{~ms}\right)$ were wittingly selected so to attain better sensitivity to the sample inhomogeneities. In our view, the sweep rates $V=\pi \mu_{0} H^{*} \cos (\omega t) / t_{\max }$, which are especially high both in the ascending $(t \simeq 0)$ and, in contrast to the exponentially decaying pulses $\left[5\right.$ 8], descending $\left(t \simeq t_{\max }\right)$ branches, have to result in the heat generation which is almost twice larger than this registered in [5 8]. In fact, numerical calculations [10] confirm that, owing to lower sweep rates, the descending brunch of the exponentially decaying pulses gives a minor contribution $\left(\simeq 13 \%\right.$, i.e. $0.6 \mathrm{~K}$ at $\left.\mu_{0} H^{*}=4 T\right)$ to the total temperature change $(\Delta T=4.55 \mathrm{~K}$ at $\left.\mu_{0} H^{*}=4 T\right)$.

However by simple heating of the sample, one could scarcely improve a sensibility. The heat also has to retain in the areas wherein this arises, i.e. in the areas with an excess of weak links [5]. Short length of magnetic pulses promises to resolve this problem in the most important stage, i.e. during a pulse. Nothing can stop the heat exchange within the HTS sample afterwards, but the "after-the-pulse" smearing of the heat distribution is expected to reduce the remanent flux contrast less considerably. Besides, to minimize the "after-the-pulse" effects, for a whole experimental cycle (magnetizing $\rightarrow$ dwelling time of $300 \mathrm{~s} \rightarrow$ measurements) the sample was immersed in a liquid nitrogen which, 


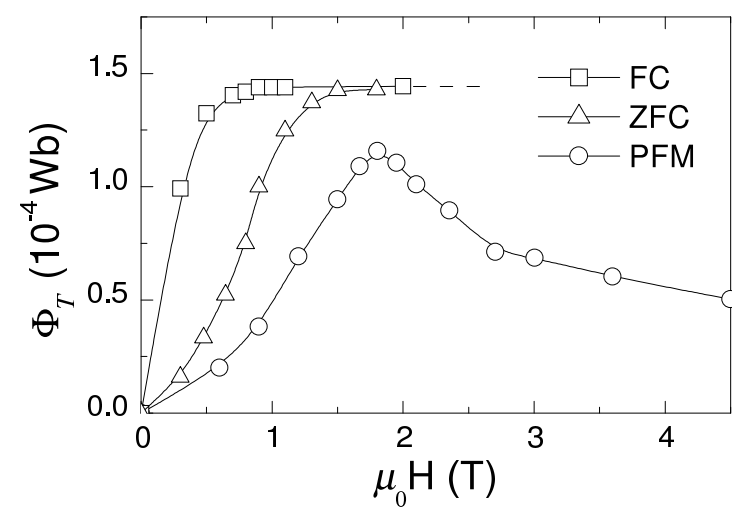

Figure 2. The applied magnetic field dependence of the total flux $\Phi_{T}$ trapped by the sample in the FC, ZFC and PFM modes $(\mathrm{T}=77 \mathrm{~K})$. In the last case, $\Phi_{T}$ is plotted vs the pulse amplitudes $\mu_{0} H^{*}$.

to our opinion, could provide a good thermal conductivity with, at least, the sample surfaces giving a main contribution to the measured flux density $B$.

The value of $B$ was mapped by the Hall sensor (Arepoc Ltd., Slovakia) which moved horizontally, $0.8 \mathrm{~mm}$ above the surface, stepwise with a pitch of $1 \mathrm{~mm}$.

\section{Results and discussion}

Before an immediate presentation of the remanent flux profiles, we have, at first, to note the following. The common-accepted value which characterizes the influence of the magnetic field on the sample ability to trap magnetic flux is the total flux $\Phi_{T}$. To calculate $\Phi_{T}$ one usually integrates the measured flux density $B$ over an entire surface $S$ where $\Phi$ is positive. Although the averaged value $\Phi_{T}=\bar{B} S$ describes the sample only as a whole, it would nevertheless be useful to compare the data obtained under our pulse conditions with those reported in references [5-8]. Figure 2 presents such dependencies for the sample magnetized in the FC, ZFC and PFM modes. Obviously, the optimal field $\mu_{0} H_{\text {opt }}=1.9 \mathrm{~T}$, where $\Phi_{T}$ in the PFM mode exhibits a maximum, corresponds to a full magnetization, i.e. the double penetration field $H_{p}=H_{o p t} / 2$.

In general, these curves are similar to those obtained elsewhere [5 7], but a certain distinction of our PFM data does seem worthy of discussion. This feature, viz., much lower ratio $R\left(H>H_{\text {opt }}\right)=\Phi_{T}^{P F M} / \Phi_{T}^{Z F C}$ of the trapped flux to its "isothermal" $(\Delta T=0)$ value (i.e. this obtained under the ZFC magnetization), is readily explained by more rapid motion of flux lines and, due to their interaction with viscous forces, more energy dissipated in the sample during a pulse. For example, $R \approx 0.65$ which was reported at $\mu_{0} H=4 T$ in Ref. [7] nearly twice exceeds similar value in Fig. 2 , $R=0.38$. Assuming that the total flux $\Phi_{T}$ is proportional to the critical current density $j_{c}$ which linearly decreases with the temperature and vanishes at $T_{c} \approx 91 K$ [13], one can roughly estimate the temperature rise as $\Delta T=(1-R) \times\left(T_{c}-77 \mathrm{~K}\right)$, i.e. $\Delta T=8.7 \mathrm{~K}$ and $\Delta T=4.9 K$ for $R=0.38$ and 0.65 , respectively. This naive approach appears, 


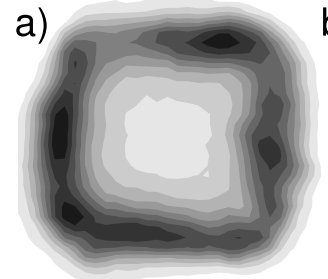

e)

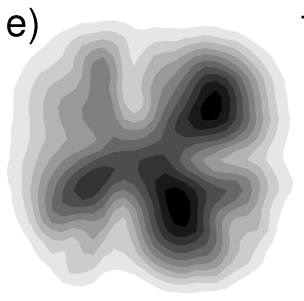

b)

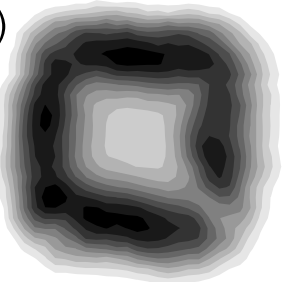

f)

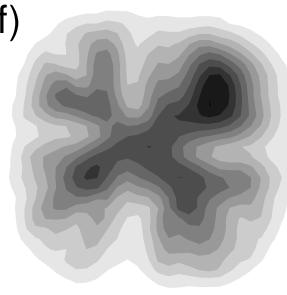

c)

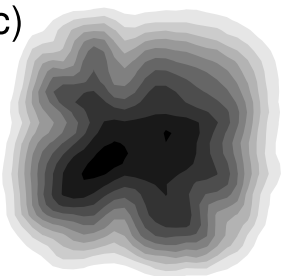

g)

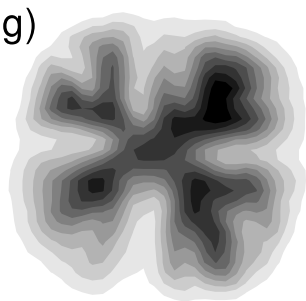

d)

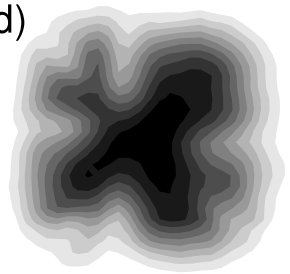

h)

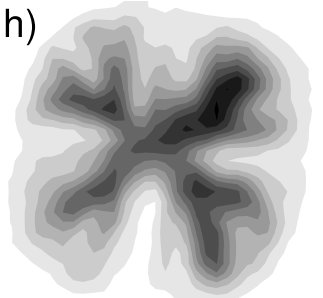

Figure 3. The trapped magnetic flux distributions for various amplitudes of the pulsed field. The figures (a)-(h) correspond to $\mathrm{T}=77 \mathrm{~K}$ and $\mu_{0} H=0.90,1.50,1.65,1.95,2.1$, 2.4, 3.0 and $4.0 \mathrm{~T}$, respectively. The different darknesses mark the $10 \%$-levels of the flux density (zero levels are white, maximum values, $B_{\max }$, are black).

nevertheless, rather accurate: the latter value is in an agreement with $\Delta T \approx 4.6 \mathrm{~K}$ calculated by authors [7] from direct measurements of flux line velocities as well as with $\Delta T=4.55 K$ given by numerical methods 10 .

However, large temperature rise $\Delta T \simeq 8.7 K$ does not yet guarantee whether the generated heat distribution reflects the HTS quality or not. The answer may be obtained from Figure 3 which shows the changes of the remanent flux distribution as $\mu_{0} H$ grows. To make this evolution more evident, we have previously normalized the flux densities $B$ for each measurement cycle to their peak value $B_{\max }$. Owing to this trick, one can easily observe how the ring-like distributions gradually transform into the "cross-wheels" which "rungs" have the form of a dovetail. If the flux maps (a) and (b) are quite typical for the ZFC procedure at $H<H_{\text {opt }}$, the high-field data, $H>H_{\text {opt }}$, seem much more interesting. At first, so large gradients $d B / d x$ and $d B / d y$, where $x$ and $y$ are the space coordinates, as those shown in Figures $3(\mathrm{e})-(\mathrm{h})$ do confirm the above suggestion that the heat transfer within the HTS bulk is relatively small. But the main result is still the following: in view of the mentioned above scenario [5], the obtained flux profiles convincingly attest that weak links are mostly distributed inside the a-GSs, while a-aGSBs are nearly free of them. Meanwhile, this arrangement is hardly noticeable under the slow magnetization. Upon a closer view, Figure 1(b) displays this difference only through minor twists of isolines (confer, for example, their pincushion distortion along the top-right a-a-GSB with the maximum in Figures $3(\mathrm{e})-(\mathrm{h}))$.

Having direct magnetic data about the distribution of weak links inside the YBCO crystal, it would be unforgivable fault to miss an opportunity of demonstration which structural defects do act as weak links. For this reason, we have cut the sample along the plane $S-S^{\prime}$ perpendicular to the a-a-GSB (see Figure 4 ) and studied this microscopic section under the polarized light. This method allows to spot subgrains (because a 

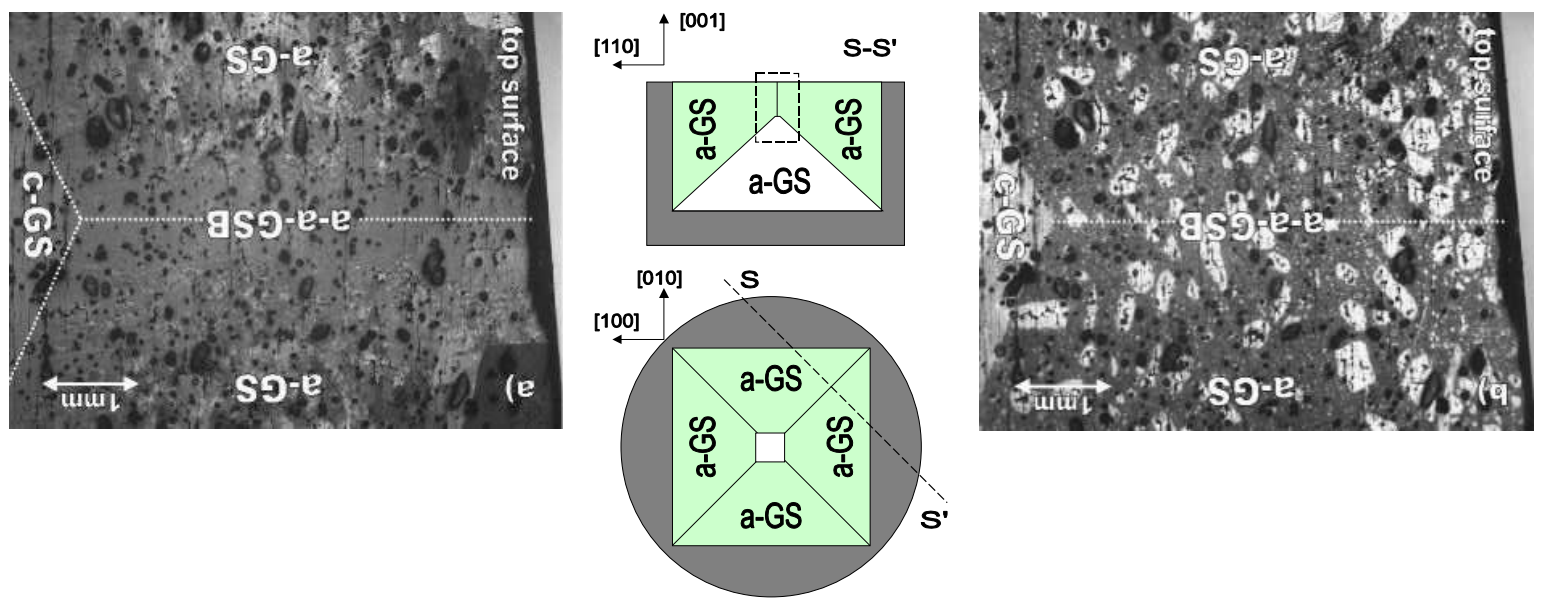

Figure 4. Polarized optical micrographs (a,b) of the same a-a-GSB fragment which corresponds to the rectangle area restricted by dashed line in the sketch (c). The image (a) shows that the a-a-SGB is surrounded by a subgrain-free band, the picture (b) illustrates the 211-density difference between the a-GSs and the c-GS (more dark regions correspond to higher 211-density).

minor misalignment yields different colors and contrasts) as well as other imperfections of the crystalline structure. Following the expectations [11], the image (a) shows the subgrain-free band (with a width of approximately $1 \mathrm{~mm}$ ) between the sample surface and the c-GS apex. It confirms that weak links in the YBCO material are mainly related to subgrain boundaries [14].

Figure $1(\mathrm{~b})$ helps to answer another important question: Why the geometric centre of the HTS sample, wherein the heat generation is minimal even at $H \gg H_{\text {opt }}$ [0, 10], does not correspond in Figures $3(\mathrm{e})-(\mathrm{h})$ to $B_{\max }$ ? In particular, this image shows that the density of the $\mathrm{Y}_{2} \mathrm{BaCuO}_{5}(211)$ inclusions, i.e. pinning centers responsible for the $j_{c}$ values, does not change across the a-a-GSB, but differs essentially at intersecting a-c-GSBs. Due to the polishing process which exposures in this place the c-GS apex (see the sketches presented in Figures 1 and 4 ) with a smaller $j_{c}$, this area can not carry a whole current that flows along a-a-GSBs. Thus, only a portion of current loops does have the X-like shape, whilst the others are closed around the GSB segments. That is the reason why the local maxima have to be exhibited by centers of these segments, but not by the geometric centre of the sample. Actually, the positions of the maxima have to move a bit closer to the crystal rims since current loops in lower planes, where the a-a-GSBs are edged out due to the c-GS expansion, also contribute to the measured flux density.

The only issue, namely, the "dovetail"-form of isolines close to the crystal edges, still remains unclear. One can presume that the isoline forks correspond to bifurcations of the a-a-GSBs, so the forks mark the points where new, secondary a-GSs are spontaneously generated. However, this question lies outside the scope of current work, it would be the subject of further studies 13 . 


\section{Conclusions}

In summary, we can conclude that the PFM does seem a promising method not only for a technical magnetization of HTS bulks, but also for their characterization: it is fast, non-destructive and, provided that appropriate parameters of magnetic pulse are used, very sensitive to minor inhomogeneities [13] of the HTS material. The main conditions which may guarantee this sensibility are the following.

- The amplitude $H^{*}$ of magnetic pulses has to much exceed the double penetration field $H_{p}$ which linearly increases with the sample size $d$ and shielding properties $\left(\sim j_{c}\right)$ of the HTS material (for example, $H_{p}$ in the $\mathrm{SmBa}_{2} \mathrm{Cu}_{3} \mathrm{O}_{7-\delta}$ crystals is twice larger than this in the $\mathrm{YBa}_{2} \mathrm{Cu}_{3} \mathrm{O}_{7-\delta}$ ones with the same size [7]).

- Except for the pulse amplitude, its duration $t_{\max }$ and the form are also responsible for high velocities $V \simeq \pi \mu_{0} H^{*} / t_{\max }$ of magnetic flux lines and, because of their interaction with viscous forces, considerable heat generation. Besides, short pulse duration provides nearly adiabatic conditions when both the heat transfer to a cooling media and this within the sample are negligible.

- The heat exchange between the sample and a cooling media has to be good enough to minimize the "after-the-pulse" smearing of the heat distribution.

Since these requirements are quite easy to satisfy, this technique seems the most suitable to explore magnetic properties of modern, nearly perfect HTS crystals. Obviously, its importance should evergrow with a further enhancement of the HTS samples to be studied.

\section{Acknowledgments}

This work was supported by the German BMBF under the project No.13N6854A3. One of the authors (A.S.) would like to thank Z.H.He and P. Diko for illuminating discussions as well as R. Müller for his continual encouragement throughout the present work. We are also indebted to M. Arnz and Ch. Schmidt for technical support.

\section{References}

[1] Murakami M 2000 Supercond. Sci. Technol. 13448

[2] Klupsch Th, Strasser Th, Habisreuther T, Gawalek W, Gruss S, May H, Palka R and Mora Serrano F J 1997 J. Appl. Phys. 823035

[3] Navau C and Sanchez C 1999 IEEE Trans. Appl. Supercond. 91610

[4] Itoh Y, Yanagi Y, Yoshikawa M, Oka T, Harada S, Sakakibara T, Yamada Y and Mizutani U 1995 Japan. J. Appl. Phys. 345574

[5] Itoh Y and Mizutani U 1996 Japan. J. Appl. Phys. 352114

[6] Mizutani U, Ikuta H, Hosokawa T, Ishihara H, Tazoe K, Oka T, Itoh Y, Yanagi Y and Yoshikawa M 2000 Supercond. Sci. Technol. 13836

[7] Ikuta H, Ishihara H, Hosokawa T, Yanagi Y, Itoh Y, Yoshikawa M, Oka T and Mizutani U 2000 Supercond. Sci. Technol. 13846

[8] Mizutani U, Oka T, Itoh Y, Yanagi Y, Yoshikawa M and Ikuta H 1998 Appl. Supercond. 6235 
[9] Sandler M, Sutter U, Koch R and Kläser M 2000 Supercond. Sci. Technol. 13841

[10] Tsuchimoto M and Morikawa K 1999 IEEE Trans. Appl. Supercond. 966

[11] Diko P 2000 Supercond. Sci. Technol. 131202

[12] Litzkendorf D, Habisreuther T, Wu M, Strasser T, Zeisberger M, Gawalek W, Helbig M and Görnert P 1998 Mat. Sci. Eng. B53 75

[13] Surzhenko A B et al (unpublished)

[14] Hedderich R, Schuster Th, Kuhn H, Geerk J, Linker G and Murakami M 1995 Appl. Phys. Lett. 663215 